\title{
Controlling Artificial Limb Movement System using EEG Signals
}

\author{
V.V. Ramalingam*, A. Pandian and R. Parivel \\ Department of Computer Science and Engineering, SRM University, Kattankulathur, Chennai - 603203, Tamil Nadu, \\ India; ramalingam.v@ktr.srmuniv.ac.in, pandian.a@ktr.srmuniv.ac.in, levirap87@gmail.com
}

\begin{abstract}
Objectives: We mainly focus the application of machine learning for artificial limb movement system using Electroencephalogram (EEG) signals. Analysis: EEG signals depict the neuronal activity happening in brain, which will be used to control the artificial limb movement system. Findings: In this paper, four classes of EEG signals were recorded from healthy subjects while performing actions such as finger open (fopen), finger close (fclose), wrist clockwise (wcw) and wrist counterclockwise (wccw) movements. The main objective of this study is to extract the statistical features from EEG signals and identify the best possible features and classify them using J48 Decision Tree algorithm. Improvements: The EEG signals are complex in nature and machine-learning approach was used to study the same. To improve the classification accuracy better feature extraction techniques might be used.
\end{abstract}

Keywords: Electroencephalogram (EEG) Signals, J48 Algorithm, Statistical Features

\section{Introduction}

Artificial limbs are a second opportunity for physically disabled as they restore the normal functioning of a person. EEG signals are complex in nature and require high level of expertise to interpret the same. These signals are direct representation of brain activity when humans perform actions. These signals when caught can be utilized to control an artificial limb but however would require a proper mechanism to map these signals into physical movement. Machine learning is the best approach to address the same. EEG signals captured from right hand wrist movements are used and statistical features are extracted from the same. From this the best features which provides highest classification accuracy is suggested for controlling the artificial limb. Previous studies in this area have used EMG (Electromyogram) signals to control the artificial limb. There are a number of disadvantages in the aforesaid approach. Firstly, acquiring EMG signals requires an invasive procedure of inserting a needle into the muscle. Besides, lot of cross talk can be heard from the adjacent muscles in performing the same. Also if there is more fat tissue deposited over the muscle, then the signal acquired may be weak. This study focuses on controlling the artificial movement through machine learning approach.

In 1 has proposed an approach to control the artificial limb using state feedback PI Controller. Wavelet coefficients were calculated from the acquired EEG signals that served as features. These features were classified using QDA classifier to move the artificial limb towards target direction and position. $\mathrm{In}^{2}$ Linear Discriminant Analysis classifier was used to classify three subsets of EEG signals (Movement Planning, Movement Execution, Steady Position). Four upper limb movements using Spatial Features were classified. Results indicate that Movement planning subset provides best classification accuracy that can be used to speed up the BCI. In $^{3}$ the velocity of both hand and elbow are studied in detail to control the upper limb movement using EEG signals. The results that were decoded using liner model for both hand and elbow movement trajectory shows that they can be used to con-

${ }^{*}$ Author for correspondence 
trol the artificial limb movement. $\operatorname{In}^{4}$ wrist movements are controlled using EEG signals. Energy and Entropy were computed to serve as features and discrete cosine transform was utilized to perform dimensionality reduction. Results show that highest accuracy is achieved while classifying discrete cosine transform features using the linear classifier. $\operatorname{In}^{5}$ the author has proposed an approach to train the patients who are about to receive the artificial limb in a virtual environment using Emotive Software Development Kit. This will prepare the patient for adapting with the artificial limb and control it efficiently using EEG signals. In ${ }^{6}$ the author has decoded the hand movement velocity from EEG signals using Multiple Linear Regression (MLR) and Kernel Ridge Regression (KRR). Results show that both low variation and high variation velocity hand movements can be decoded effectively using MLR and KRR. $\operatorname{In}^{7}$ the target position of shoulder and elbow are estimated with the help of EEG signals, as they are vital to perform day to activities with the artificial limb. Root Mean Square (RMS) method was used to extract the features from EEG signals and classified using neural networks. Different level of accuracy was obtained across various day-to-day tasks. $\operatorname{In}^{8}$ has classified the EEG signals from 14 channels for upper limb movement using spiking neural networks (SSN). The signals were initially encoded using Ben's Spike Algorithm and then fed into a 3D mapper called NeuCube. Classification was performed both with and without NeuCube. In ${ }^{9}$ the author has studied the EEG signals during the movement of right and left elbow joints. Fast Fourier Transfer was used to perform the same. Artificial neural network was utilized to identify whether the subject has actually performed an elbow movement and the classification accuracy was measured. $\operatorname{In}^{10}$ has studied the effects of classifying EEG signals using wavelet features. Classifiers- Naive Bayes, Multi layered Perceptron and Support Vector Machine was used to classify the computed features. A result shows that Daubechies wavelet of order 4 serves as high quality feature. $\mathrm{In}^{\underline{11}}$ the author has extracted spectral and temporal features from EEG signals while the subjects performed three different types of hand movements and classified the same using Linear Discriminant Analysis (LDA). Feature reduction was carried out using Sequential Forward Selection (SFS) and Principal Component Analysis (PCA). Results showed that spectral features produced higher accuracy when compared to temporal features. $\operatorname{In}^{12}$ the author has classified the EEG signals for upper limb analytical movement using Bayesian classifier.
Alpha and Beta bands were considered as features. Feature selection was carried out using genetic algorithm. $\mathrm{In}^{1 \underline{13}}$ the author has classified various movements such as right hand, left hand, tongue, and right foot movements using EEG signals. In ${ }^{14}$ has utilized binary SVM classifier and classified the automatic sleep staging using EEG signals. SVM based Recursive Feature Elimination (SVM-RFE) technique was used for feature selection. In $\frac{15}{5}$ the author has discussed a non-invasive procedure for inferring hand movements from MEG, EEG and intracranial EEG signals. This serves as a hope for BCI controlled rehabilitation. In ${ }^{16}$ the author has classified EEG signals for motor imaginary tasks. The EEGs were decayed into a series of frequency bands which is represented by envelop of oscillatory movement. This structure the spatial patterns for a given electrode montage at a time-frequency matrix. The signals were classified using 10 -fold cross-validation with convincing accuracy. $\operatorname{In} \frac{17}{7}$ proposed EEG based BCI system used to identify the different mental tasks with the help of geneatative independent component analysis model. Bayes rule was used for classification. Results suggest that using gICA alone is powerful enough to produce good performance. ICA as a preprocessing step for power spectral density SVM classifiers also tends to improve the performance although giving the same performance of gICA. In ${ }^{18}$ has demonstrated the benefits of performing dimensionality reduction using filters and wrappers. SVM and MLP classification was used to classify the features. Results show that dimensionality reduction aids accuracy and improves the performance of classifier. $\operatorname{In} \underline{19}$ feature extraction from EEG signals was carried out using computing Lyapunov exponents, power levels of PSD values obtained by the eigenvector methods and wavelet coefficients. Dimensionality reduction was carried out using Statistics. Levenberg-Marquardt algorithm was used for classification. Results conclude that the proposed MLPNN has effective in detecting the electroencephalographic changes. $\mathrm{In}^{20}$ the author has compared EEG and EMG signals in controlling artificial limb movements. Statistical features were computed to serve as input and the same was classified using C4.5 algorithm. Results show that EEG signals are more effective in controlling artificial limb than EMG signals. $\operatorname{In}^{21}$ the author has considered 8 classes of hand movements and performed feature extraction with Histogram and Time series analysis using Auto Regressive (AR) model and Mean frequency was considered a features. The author concluded that histogram servers as better feature. $\mathrm{In}^{22}$ the author has developed a 
novel spatial filtering method for extracting the feature information underlying the event-related de-synchronization and synchronization (ERD/ERS) during complex motor imagery of lower limb action. Results conclude that WPICA-based technique, which is better than the traditional ICA. $\mathrm{In}^{23}$ the author reported that the Clonal Selection Classification Algorithm with statistical features was used to classify four different hand movements. The paper is organized as follows, the Section 2, deals with system architecture and data acquisition. In Section 2.1, Feature extraction concepts. In Section 2.2, Feature selection concepts. Result and discussion are narrated and the applications of the decision tree trained by EEG signals are presented in Section 3. Finally, the conclusions related to the present study are emphasized in Section 4.

\section{Materials and Methods}

The EEG signals were extracted from 27 different and healthy subjects with two minutes of gap between each sequence to avoid muscle fatigue. The subjects were allowed to sit comfortably on an adjustable chair and the electrodes (C3, C4, CZ, FZ and PZ) were placed on their scalp, according to the order is shown in Figure 1.

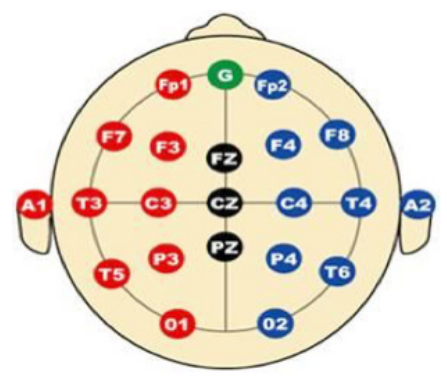

Figure 1. EEG electrode placement system.

Sufficient amount of conductive gel was applied to the scalp in prior. Four different classes of movements such as fopen (Finger open), fclose (Finger close), wcw (wrist clockwise) and wccw (wrist counterclockwise) were asked to be performed. EEG signals were acquired from the electrodes with the help of Electroencephalogram device (RMS kit) with the frequency ranges from 8 to $13 \mathrm{~Hz}$. The signal length was kept as 1024 for all channels in common. Only right hand signals were acquired as majority of the worlds population attribute to right hand habituation. The complete architecture of the present study is shown in Figure 2.

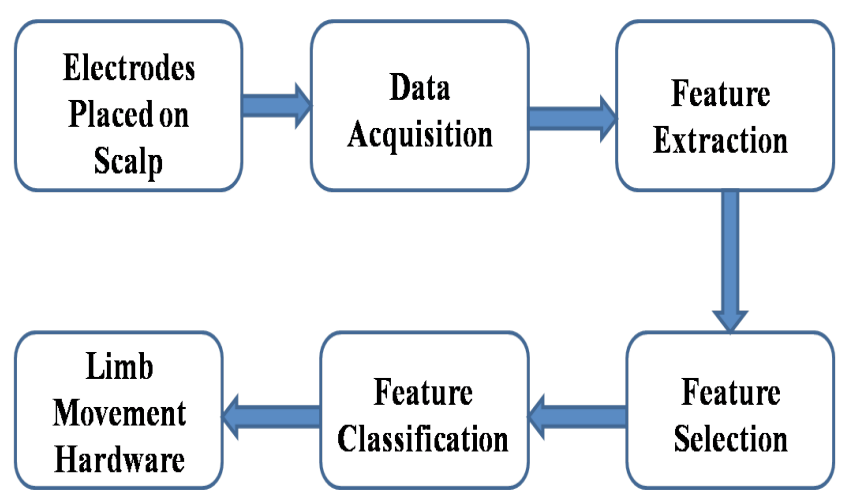

Figure 2. System architecture for artificial limb control system.

\subsection{Feature Extraction}

From the acquired EEG signals parameters such as standard deviation, minimum, maximum, sum, mean, median, mode, kurtosis, skewness, standard error, variance, count and range were extracted to serve as statistical features. The detailed process is explained below.

Each movement class performed by the subject will result in one signal file per channel. For example, Finger open movement performed by a subject will create 4 signal files in total (one file per channel C3, C4, CZ, FZ, PZ this will be in the format of a text file. The total number of files for each class with respect to the five channels is given below.

The statistical feature for each channel is extracted separately. This is achieved with the help of excel Macros. Therefore, each channel will have 108 records comprising all the four movements. A sample macro that was used to extract the statistical features of finger close class for $\mathrm{C} 3$ channel is given in Table 1 .

The statistical feature for each channel is extracted separately. This is achieved with the help of excel Macros. Therefore, each channels dataset will have 108 records comprising all the four movements. A sample macro that was used to extract the statistical features of finger close class for $\mathrm{C} 3$ channel is given below.

\section{Sample Macro:}

Sub Macro()

For $\mathrm{i}=1$ To 27

Range("A" \& i \& " ").Select

Workbooks.OpenText Filename:=

"C: $\mid$ Users $\backslash$ Local $\backslash$ Desktop $\backslash$ final_eeg_signal $\backslash$ finger_ closelc3\c3” \& i \& “.txt”, Origin:= 
xlMSDOS, StartRow:=1, DataType:=xlDelimited, TextQualifier:=xlDoubleQuote _

, ConsecutiveDelimiter:=False, Tab:=True, Semicolon:=False, Comma: =

False, Space:=False, Other:=False, FieldInfo: $=\operatorname{Array}(1$, $1)$,

TrailingMinusNumbers:=True

Application.Run "ATPVBAEN.XLA!Descr", ActiveSheet.Range(“\$A:\$A”), “”, “C”

, False, True

Range(“B3:B15”).Select

Selection.Copy

Range("E3").Select

Selection.PasteSpecial Paste:=xlPasteAll,

Operation:=xlNone, SkipBlanks:=

False, Transpose:=True

Application.CutCopyMode $=$ False

Selection.Copy

ActiveWindow.Close

ActiveSheet.Range("A" \& i \& "').Select

ActiveSheet.Paste

Next i

End Sub

Table 1. Total signal files per channel for each class

\begin{tabular}{|l|l|l|l|l|}
\hline Channel & fopen & fclose & wcw & wccw \\
\hline C3 & 27 & 27 & 27 & 27 \\
\hline C4 & 27 & 27 & 27 & 27 \\
\hline CZ & 27 & 27 & 27 & 27 \\
\hline FZ & 27 & 27 & 27 & 27 \\
\hline PZ & 27 & 27 & 27 & 27 \\
\hline
\end{tabular}

This macro takes each signal file, computes statistical features using the data analysis package available in excel and saves the output to a excel file. Thus all signals are converted into statistical features.

Post Statistical Feature extraction, dataset from each channel is classified using C4.5 Decision Tree algorithm. The classification performed using $\mathrm{C} 4.5$ for each channel suggests that the channel $\mathrm{C} 4$ provides more Classification accuracy. Hence we will be considering channel $\mathrm{C} 4$ for further study. This is evident from Figure 3.

\subsection{Feature Selection}

For feature selection process, only channel $\mathrm{C} 4$ will be considered as it provides the highest accuracy when com- pared to other channels. The feature selection process is achieved by constructing a decision tree with the help of C4.5 algorithm. Initially all the attributes extracted are considered to construct the decision tree. From the constructed tree it is evident that only the attributes Median, Maximum, Mean, Skewness, Kurtosis, Range, Standard Error, Minimum has significance in the classification (Figure 4).

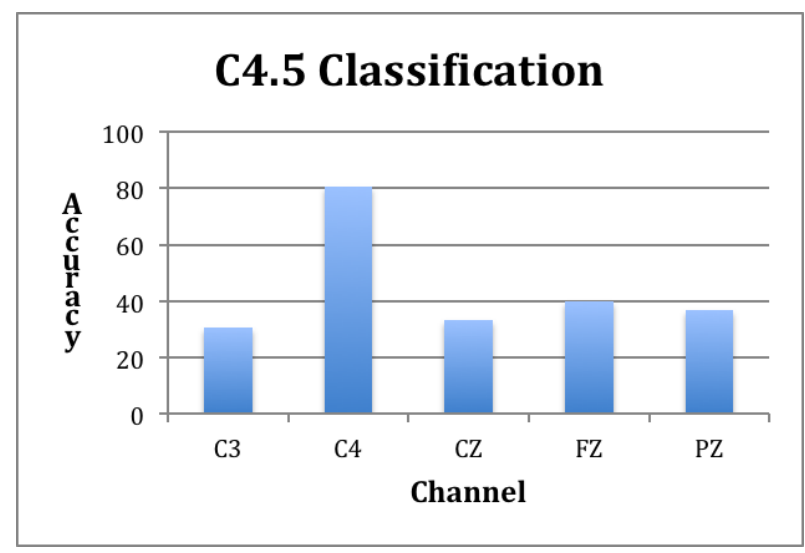

Figure 3. Classification accuracy achieved over various channels.

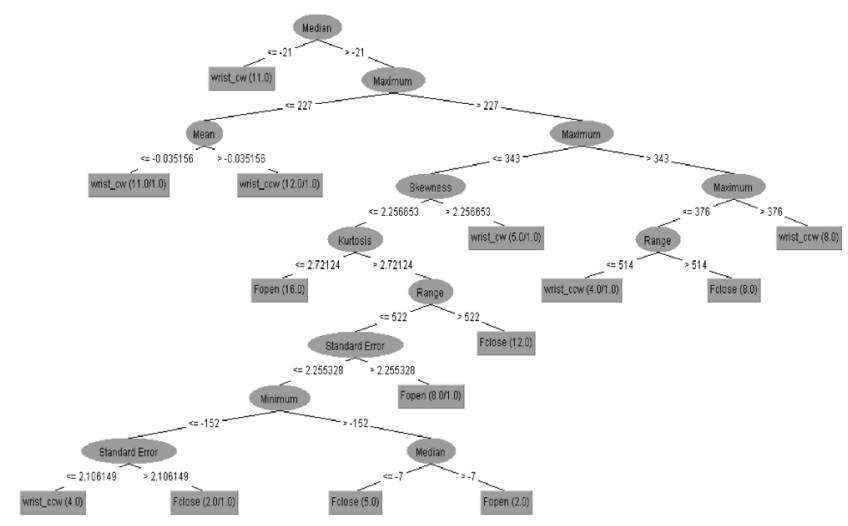

Figure 4. Decision tree using statistical features.

The next step in feature selection is to perform dimensionality reduction to minimize the classifier complexity. The 8 attributes that were identified as essential ones are added one by one to the decision tree and the classification accuracy is recorded for validation. This process begins with the attribute Median and ends with Minimum.

The results of dimensionality reduction indicate that the attributes Median, Mean and Maximum provide the highest classification accuracy of $80.55 \%$. Adding further dependent attributes reduces the accuracy to $78.1 \%$ is shown in Figure 5. 


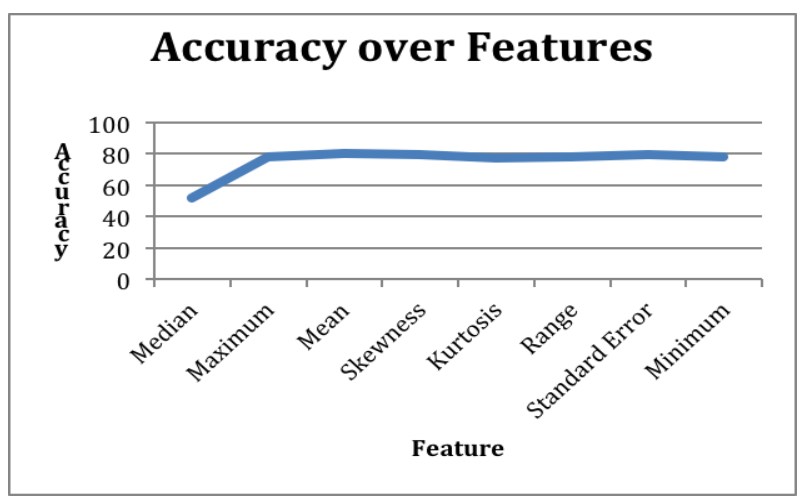

Figure 5. Features vs classification accuracy.

\section{Results and Discussion}

In section 2, the statistical feature extraction process from the acquired EEG signals is discussed in detail. Classification performed over the extracted features using $\mathrm{C} 4.5$ algorithm suggested that channel $\mathrm{C} 4$ provided more accuracy when compared to other channels. In section 3, feature selection process denoted that the features Mean, Median and Maximum provides the highest classification accuracy of $80.55 \%$.

Minimum number of Objects and Confidence factor are two import parameters that affect the classification accuracy of C4.5 Decision Tree algorithm is shown in Figure 6. In our classification, with a default confidence 0.2 , the minimum number of objects was varied from 1 to 27 and the accuracy was measured. The accuracy reduces when the number of objects goes beyond 3 (80.55\%). Hence the number of objects was fixed as 3 .

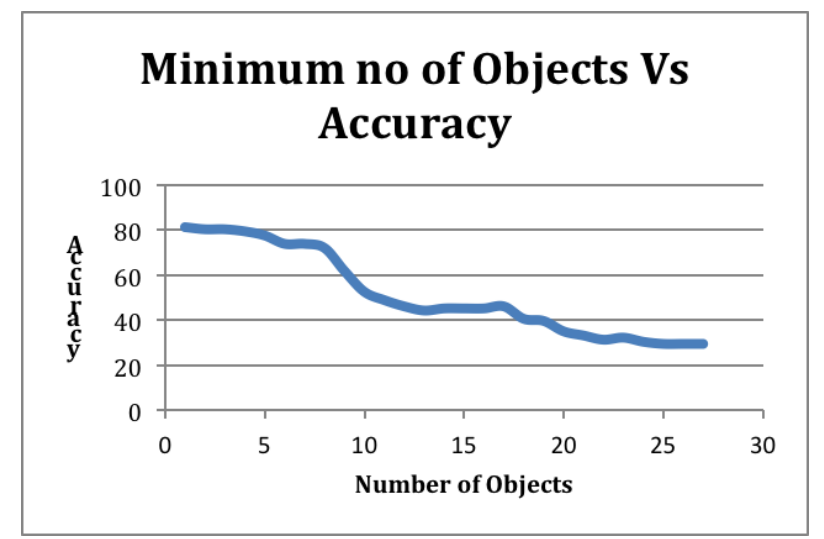

Figure 6. Minimum no. of objects vs classification accuracy.

With the number of objects fixed to 3, the confidence was varied from 0.1 to 1 in steps of 0.1 . An accuracy of
$81.48 \%$ was achieved when the confidence factor was set to 0.1 is shown in Figure 7 .

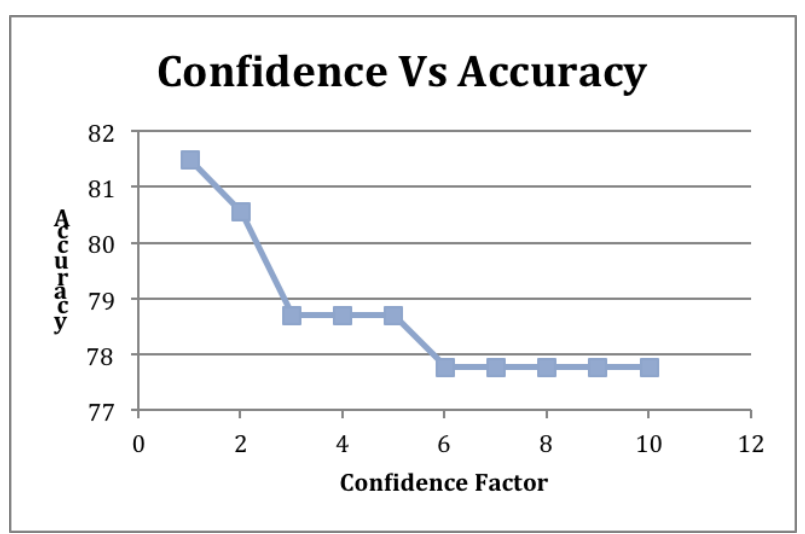

Figure 7. Confidence factor vs classification accuracy.

Hence we can conclude that the classifier attains maximum classification accuracy when the confidence factor is set to 0.1 and the minimum number of objects is kept as 3 .

Table 2. Confusion matrix

\begin{tabular}{|l|l|l|l|l|}
\hline & fclose & fopen & wccw & wcw \\
\hline fclose & 23 & 3 & 0 & 1 \\
\hline fopen & 1 & 23 & 2 & 1 \\
\hline wccw & 4 & 4 & 19 & 0 \\
\hline wcw & 1 & 1 & 2 & 23 \\
\hline
\end{tabular}

Table 3. Summary of classifier results

\begin{tabular}{|l|l|}
\hline Correctly Classified Instances & $\mathbf{8 1 . 4 8}$ \\
\hline Incorrectly Classified Instances & 18.51 \\
\hline Kappa statistic & 0.75 \\
\hline Mean absolute error & 0.12 \\
\hline Root mean squared error & 0.28 \\
\hline Relative absolute error & $32.77 \%$ \\
\hline Root relative squared error & $65.63 \%$ \\
\hline Total Number of Instances & 108 \\
\hline
\end{tabular}

The Classification accuracy can be presented in the form of confusion matrix and the same is show in Table 2. The diagonal elements of the matrix represent the correctly classified instances and the non-diagonal elements represent the incorrect classification instances. The first row in the confusion matrix details the how the classifier has performed over classifying "fclose" class. Each 
Table 4. Detailed accuracy by class

\begin{tabular}{|l|l|l|l|l|l|l|}
\hline $\begin{array}{l}\text { TP } \\
\text { Rate }\end{array}$ & $\begin{array}{l}\text { FP } \\
\text { Rate }\end{array}$ & Precision & Recall & F-Measure & $\begin{array}{l}\text { ROC } \\
\text { Area }\end{array}$ & Class \\
\hline 0.852 & 0.074 & 0.793 & 0.852 & 0.821 & 0.898 & fclose \\
\hline 0.852 & 0.099 & 0.742 & 0.852 & 0.793 & 0.894 & fopen \\
\hline 0.704 & 0.049 & 0.826 & 0.704 & 0.76 & 0.85 & wccw \\
\hline 0.852 & 0.025 & 0.92 & 0.852 & 0.885 & 0.901 & wcw \\
\hline
\end{tabular}

column header represents the result of classifier on classifying the given instance. So, in this case, fclose has been correctly classified in 23 instances and incorrectly classified as "fopen" in 3 instances and "wcw" in 1 instance. Similarly, second, third and fourth rows can be interpreted of each class.

The Summary of classifier performance is shown in Table 3. The classification accuracy achieved is $81.48 \%$. Kappa statistics is a measure of agreement between two categories of data set such as the predicted and the observed. Mean absolute error (MAE) is a quantity used to measure how close predictions are to the eventual outcomes. Root means square is the difference between values predicted by a model and the values actually observed from the environment that is being modeled. The Detailed accuracy achieved by Decision Tree algorithm is given in Table 4 .

\section{Conclusion}

In this paper, four classes of right hand movements were considered. The descriptive statistical features were computed from EEG signals. Feature selection was carried out to reduce the classifier complexity and an accuracy of 80.55\% was achieved using C4.5 decision tree algorithm. Further tweaking the parameters, the classification accuracy of $81.48 \%$ was achieved. Thus, EEG signals can be used to control artificial limb movements system using C4.5 Decision Tree Algorithm.

\section{References}

1. Roy R, Konar A, Tibarewala DN. EEG driven artificial limb control using state feedback PI controller. IEEE Students Conference on Electrical, Electronics and Computer Science; 2012. p. 1-5.

2. Caracillo RC, Castro MCF. Classification of executed upper limb movements by means of EEG. 2013 ISSNIP Biosignals and Biorobotics Conference (BRC); 2013. p. 1-6.
3. Kim JH, Chavarriaga R, Millan JDR, Lee SW. Three dimensional upper limb movement decoding from EEG signals. IEEE International Winter Workshop on Brain-Computer Interface (BCI); 2013. p. 109-11.

4. Ghani F, Gaur B, Varshney S, Farooq O, Khan YU. Detection of wrist movement using EEG signal for brain machine interface. IEEE International Conference on Technology, Informatics, Management, Engineering and Environment; 2013. p. 5-8.

5. Nogueira KL, Souza E, Lamounier A. Architecture for controlling upper limb prosthesis using brainwaves. 2013 International Conference on Virtual Rehabilitation (ICVR),; 2013. p. 178-9.

6. Jeong HK, Felix B, Seong WL. Reconstruction of hand movements from EEG signals based on non-linear regression. IEEE-Brain-Computer Interface (BCI), 2014 International Winter Workshop; 2014. p. 1-3.

7. Kazuo K, Yoshiaki H. Task estimation of upper-limb using EEG and EMG signals. IEEE/ASME International Conference on Advanced Intelligent Mechatronics (AIM); 2014. p. 548-53.

8. Jin H, Zeng GH, Yi XC, Nikola K, Nathan S. EEG-based classification of upper-limb ADL using SNN for active robotic rehabilitation. EEE RAS and EMBS International Conference on Biomedical Robotics and Biomechatronics (BioRob); 2014.

9. Yoshiaki H, Kazuo K. A study of features of EEG signals during upper-limb motion. IEEE International Conference on Advanced Intelligent Mechatronics (AIM); 2015. p. 943-6.

10. 10. Rajdeep C, Tathagata B, Debarshi K S. Effects of Wavelets on Quality of Features in Motor Imagery EEG Signal Classification. IEEE WiSPNET conference, 2016, pp. 1346-1350.

11. Mads J, Imran KN, Kim D, Ernest NK. Detecting and classifying three different hand movement types through electroencephalography recordings for neuro rehabilitation. Medical Bio Engineering Computing. 2016; 54:1491-501.

12. Jaime I, Serrano JI, Castillo MD. Predictive classification of self paced upper limb analytical movements with EEG. International Federation for Medical and Biological Engineering. 2015; 53(11):1201-10. 
13. Valerie M, Ou B, Stephen F, Peter L, Mark H. Classifying EEG signals preceding right hand, left hand, tongue, and right foot movements and motor imageries. Clinical Neurophysiology. 2008; 119(11):2570-8.

14. Koley B, Dey D. An ensemble system for automatic sleep stage classification using single channel EEG signal. Computers in Biology and Medicine. 2012; 42:1186-95.

15. Jerbi K Vidal JR, Mattout. J, Maby E. Inferring hand movement kinematics from MEG, EEG and intracranial EEG: From brain-machine interfaces to motor rehabilitation. IRBM. 2011; 32:8-18.

16. Tao W, Jie D, Bin H. Classifying EEG-based motor imagery tasks by means of time-frequency synthesized spatial patterns. Clinical Neurophysiology. 2004; 115:2744-53.

17. Silvia C, David B. EEG classification using generative independent component analysis. Neurocomputing. 2006; 69:769-77.

18. Diego AE, Noelia SM, Amparo AB, Moret-Bonillo V. Reducing dimensionality in a database of sleep EEG arousals. Expert Systems with Applications. 2011; 38:7746-54.
19. Ubeyli ED. Statistics over features: EEG signals analysis. Computers in Biology and Medicine. 2009; 39:733-41.

20. Ramalingam VV, Mohan S, Sugumaran V. A comparison of EMG and EEG signals for prostheses control using decision tree. International Journal of Research in Computer Applications and Information Technology. 2013; 1(1):1-8.

21. Aishwarya R, Prabhu M, Sumithra G, Anusiya M. Feature extraction for EMG based prostheses control. ICTACT Journal on Soft Computing. 2013; 3(2):472-7.

22. Zhou Z, Wan B. Wavelet packet-based independent component analysis for feature extraction from motor imagery EEG of complex movements. Clinical Neurophysiology. 2012; 123:1779-88.

23. Ramalingam VV, Mohan S, Sugumaran V. Prosthetic arm control using Clonal Selection Classification Algorithm (CSCA) - A statistical learning approach. Indian Journal of Science and Technology. 2016; 9(16):1-8. 